\title{
The effect of presentation method on short-term recall of CCC trigrams
}

\author{
D. MARCER \\ UNIVERSITY OF SOUTHAMPTON, ENGLAND
}

Short-term recall of single trigrams was measured in a Peterson (1959) type situation comparing three presentation methods, one auditory and two visual. In the visual conditions the stimuli were presented grouped as a three letter unit for $1 \mathrm{sec}$, or spaced letter by letter within $1 \mathrm{sec}$. In the auditory condition $S s$ heard the stimulus within $1 \mathrm{sec}$. Most efficient recall was in the visual grouped condition, with the visual spaced presentation giving recall approximating to the auditory condition.

Melton (1963) suggests that the number of Millerian (1956) chunks rather than the number of physical elements within the stimulus is the critical determinant of the short-term retention curve. Using stimuli of one, two, three, four, or five consonants Melton (1963) obtained a family of retention curves which showed retention decreased with the number of items in the stimulus. Despite certain methodological objections, since investigated by Houston (1965), these findings support Melton's explanation in terms of intra-unit interference.

A comparison of Melton's three consonant slope with those obtained by Peterson \& Peterson (1959) shows, however, much less forgetting occurred in Melton's study. As the Petersons used auditory presentation it seems reasonable to assume that their Ss would have far less chance to code the stimulus as a single chunk than would Melton's, who saw all three letters together.

The experiment reported here investigates an alternative visual situation, that is, when each of the letters is seen separately.

\section{Method}

Ss were 36 undergraduates randomly assigned to three groups of 10 plus three smaller control conditions. Stimuli were 20 CCC trigrams, with a mean association value of $25 \%$ (Witmer, 1935). For both visual conditions stimuli were printed in capital letters on a tape which moved behind an aperture. The auditory material was recorded on magnetic tape. Figure 1 represents the experimental situation.

The metronome was set at the highest speed at which $S$ could count backwards without errors. This speed was determined for each $S$ prior to the experiment. Each $\mathrm{S}$ wes given five practice items before the experimental session.

Three control groups were also tested for recall after zero and $18 \mathrm{sec}$ unoccupied time.

Resulis and Discussion

The data for each conditions were analyzed for recall of correct units, ordered recall, item recall, and position recall. This method of analysis is discussed in detail by Wickelgren (1965a).

Unit recall. The recall of control groups under all conditions was $100 \%$. The recall of complete units for V.G., V.S., and A presentation was $44.5 \%, 18.5 \%$, and 27.0\%, respectively. Analysis of variance shows V.G. scores to be significantly greater than the other two conditions $(p<.01)$. The difference between V.S. and A groups does not reach the $5 \%$ significance level.

Item recall. The item recall error rates for V.G.. V.S., and $A$ presentation were $34 \%, 45 \%$, and $44.5 \%$, respectively. Again the V.G. condition gave better recall than the other two conditions $(p<.01)$ which are not significantly different from each other.

Position recall. The position recall error rates for V.G., V.S., and A presentation were $10.15 \%, 26 \%$, and $13.17 \%$, respectively. The V.S. error rate is significantly greater than the other two conditions $(p<.001)$ which are not significantly different from each other.

To try to account for these findings requires a consideration of the task $S$ is faced with in the experimental situation. In the V.G. condition three letters are seen together for 1 sec. $S$ thus has the optimal conditions for coding the three letters as a single

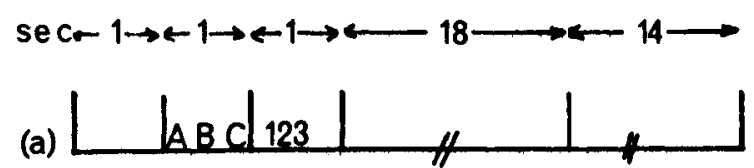

(b)
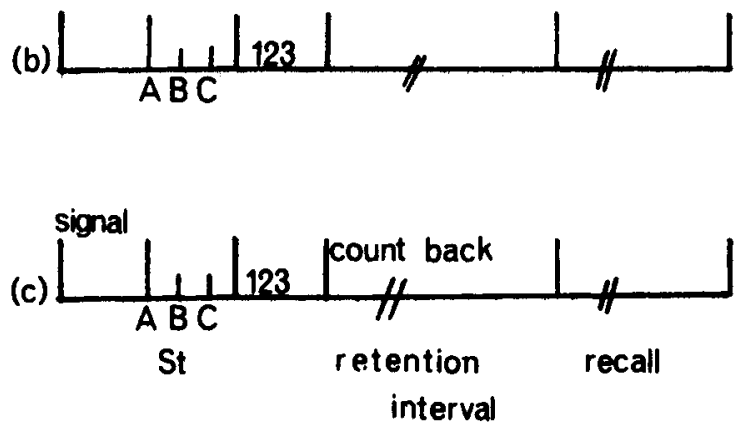

Fig. 1. Experimental situation for (a) Visual Grouped (V.G.), (b) Visual Spaced (V.S.) and (c) Auditory (A) presentation methods. In the last two conditions each letter is presented alone for $1 / 3$ sec. 


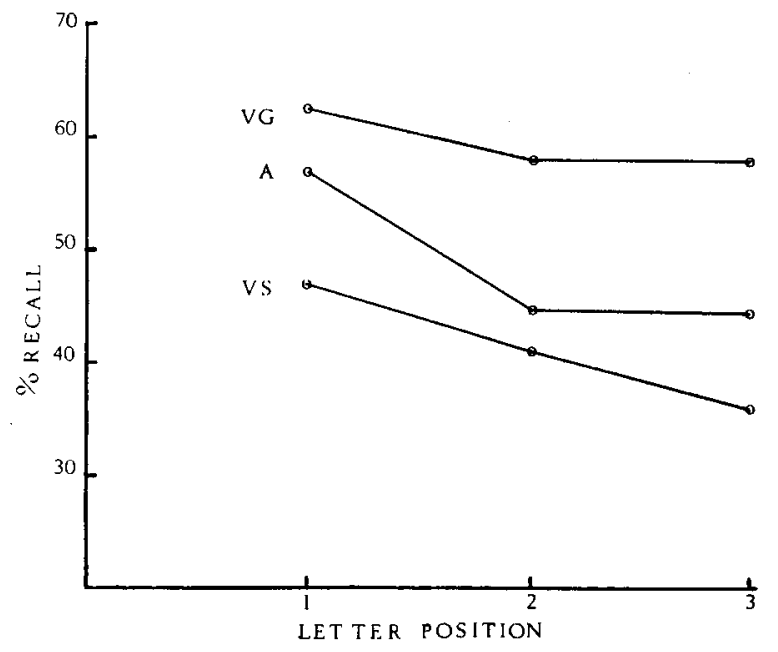

Fig. 2. Frequency of correct recall for each letter position.

chunk. If the material is such that this is not an appropriate strategy, then $S$ may choose to code it as three separate chunks. Should the latter strategy be adopted, the presence of three items simultaneously gives $S$ the chance to process each one equally. By presenting letters separately, however, the possibility of single chunking seems to be reduced to a minimum in that, until the last item is presented, $S$ has no way of integrating the items into a single chunk. Thus $S$ is only able to process the stimulus as a chunk for a maximum of $1 / 3 \mathrm{sec}$. It seems reasonable to assume that this method forces $S$ to code the material as three chunks much more than does the grouped presentation method. In this case, unlike the grouped situation, the available time for processing each item is not the same. The first letter is available for a maximum of $1 \mathrm{sec}$, the second for $2 / 3 \mathrm{sec}$, and the last $1 / 3$ sec. To examine this suggestion further, the proportion of letters correct in each position for the three conditions was calculated.

Analysis of variance for each presentation method shows letter position to be significant $(p<.025)$ in the V.S. and A conditions, but not in the V.G, condition.

At this stage it is tempting to assume that these differences can be explained simply in terms of chunking and intra-unit interference. When three letters are presented in a form which enables $S$ to code them as a discrete unit or chunk, this is done. When the letters are presented individually, $S$ processes them as three chunks, thus explaining the superior recall in V.G. condition. Further, as the letters in the V.G. condition are integrated into one chunk, the second and third letters are equally well stored. In the other two conditions, however, the recall of each item might vary with the time available to process it. Figure 2 supports this interpretation.

Position recall error rate can also be interpreted in this way. The more chunks a stimulus contains, the more interference between items would be expected, resulting in a loss of "order information." In the case of the V.S. condition position recall error rate is significantly greater than the V.G. condition $(p<.001)$. The position recall error rate for the $A$ condition is also greater than that for the V.G. condition, but the difference does not reach the usually accepted significance level.

Much evidence (e.g., Wickelgren, 1965b) has been produced to suggest recall errors in STM are of an acoustic nature. It seems probable that when three letters are integrated into a single chunk the individual letters may lose their acoustic identity. One might expect, therefore, to find fewer acoustic confusions in the V.G. condition where chunking is assumed. Using the vowel sound confusion matrix suggested by Wickelgren $(1965 \mathrm{~b})$, the same-sound intrusions for V.G., V.S., and A conditions were found to be $56.2 \%$. $45.8 \%$, and $58.6 \%$. While all three values are greatly above chance expectation ( $p<.001$ ), there is no significant difference between the three conditions.

While this last finding is negative, it is clear that V.S. and A presentation of material give results which have more in common with each other than with the V.G. condition. An explanation in terms of chunking and intra-unit interference seems attractive.

\section{References}

Houston, J. P. Short term retention or verbal units with equated degrees of learning. J. exp. Psychol., 1965, 70, 75-78.

Melton, A. W. Implications of short-term memory for a general theory of memory. J. verbal Learn. verbal Behav., 1963, 2, 34-39.

Miller, G. A. The magical number seven, plus or minus two: Some Some limits on our capacity for processing information. Psychol. Rev., 1956, 63, 81-97.

Peterson, L. R., \& Peterson, M. J. Short-term retention of individual verbal items. J. exp. Psychol., 1959, 58, 193-198.

Wickelgren, W. A. Acoustic similarity and retroactive interference in short-term memory. J. verbal Learn. verbal Behav., 1965a, 4, 53-61.

Wickelgren, W. A. Acoustic similarity and intrusion errors in shortterm memory. J. exp. Psychol., 1965b, 70, 102-108.

Witmer, L. R. The association-value of three-place consonant syllables. $J$. genet. Psychol., 1935, 47, 337-360. 